\title{
Study on Integrative Development of Regional Tourism of Nanchang-Fuzhou

\author{
XIONG Wen-ping
}

Nanchang Normal University, Nanchang Jiangxi, China, 330029

Icshxwp@126.com

Key words: Nanchang-Fuzhou; tourism integration; industry; integration; brand

\begin{abstract}
This paper analyzes the problems existing in promotion of integrative development of Nanchang-Fuzhou based on the advantage conditions of exploring tourism integration of NanchangFuzhou and proposes countermeasures for integrative development of Nanchang-Fuzhou.
\end{abstract}

The tourism industry serves as a pillar industry or an important industry of other strategic positioning in many countries and regions and develops rapidly in the wave of economy integration. China's regional tourism cooperation appears rapid development. It started since early 1990s, and entered regional competition phase from scenic spots competition, line competition and city competition up to the first ten years of the 21st century [1]. Competition strategies of tourism are transferring from previous pure price competition strategy and current quality competition strategy to cultural competition strategy in the future, and competitive mode of tourism also transfers from single skill competition to strategic competition and image competition [2]. Constructing regional large culture and seeking regional grand coalition becomes the fundamental strategy in competition of current era [3]. As for tourism industry, development of regional tourism is subject to the region's ability to carry out regional combination under the condition of current market economy, i.e. depending on whether this region can break away from its narrow circle to develop tourism industry and realize "resource sharing" and "market sharing" in the region [4]. Seeing from static perspective, tourism integration refers to all kinds of elements in tourism industry of different countries and regions within a region, including travelers, tourism products, tourism funds, tourism techniques and human resources of tourism, etc., which can get rational distribution and full circulation within the region and form the state of mutual dependency, advanced division, complementary advantages and resources sharing; seeing from dynamic perspective, it means the gradual course to reach tourism alliance (union) for combination of tourism field in different countries and regions for development of regional tourism and abiding by economic development laws of regional economy[5]. Therefore, sound development of tourism integration of Nanchang-Fuzhou is of significant importance for development of bilateral tourism industry.

Tourism has become one of the industries in global economy with largest development scale, and been maintaining powerful development momentum. The advantageous features of tourism such as sustainable development, strong industry relevancy, optimized industry structure and promoting employment, etc. make it play a key role in regional economic and social development [6]. Nanchang City and Fuzhou City cover an area of 26219 square kilometers with a total of 20 counties and districts. In 2015, the governments of Nanchang City and Fuzhou City signed a Cooperation Agreement on Further Promotion of Development of Nanchang-Fuzhou Linkage respectively,which determined to advance Nanchang-Fuzhou integration strategy in both Nanchang city and Fuzhou city and took promoting industrial integration development of both cities as one of main targets of realizing Nanchang-Fuzhou integration. Constructing integration of Nanchang-Fuzhou tourism industry integration will contribute to complementary advantages of tourism resources in both places and promote Nanchang-Fuzhou industrial development, thus simulating economic development towards to economic corridor of Xiangtang-Putian. Tourism covers many sectors and industries, involving many aspects. So, promoting integration development of tourism holds an important position in integration development of Nanchang-Fuzhou. This paper 
analyzes the problems existing in promotion of integrative development of Nanchang-Fuzhou based on the advantage conditions of exploring tourism integration of Nanchang- Fuzhou and proposes countermeasures for integrative development of Nanchang-Fuzhou, hoping to enrich research on this issue in theoretical research and expecting to make it of consult and reference to tour development in integration for this area and similar regions with regard to decisions.

\section{Superiority conditions to integrative development of tourism of Nanchang-Fuzhou}

\section{There are great differences in tourism resources of both Nanchang and Fuzhou, which is highly complementary.}

Both Nanchang and Fuzhou have their own typical characteristics in tourism resources. Advantages of "red" tourism resources are highlighted in Nanchang tourism resources. Nanchang is the place where army flag of the Chinese People's Liberation Army rises and birthplace for the Chinese People's Liberation Army. It has numerous red tourism resources, such as Memorial of the August 1 Nanchang Uprising and Nanchang Uprising monument on August 1, etc. Nanchang City has Xiaoping Trail, where Deng Xiaoping, "the architect of China's reforms", was delegated to local government in Jiangxi and was pregnant with the reform and opening up idea for China. Nanchang has a long history and is a famous historic and cultural city in China. It has historical relics such as Tengwang Pavilion and Wanshou Palace, etc., among which, Tengwang Pavilion is known as three famous towers, including famous Buddhism \& Taoism temples. Nanchang has urban wetland parks such as Yaohu Lake, Aixi Lake and Qingshan Lake, etc. and playgrounds such as Wanda Mall, etc. Fuzhou is the home to famous talents, in which numerous famous masters such as Tang Xianzu, Wang Anshi, Zeng Gong and Lu Jiuyuan, etc. were born, it has rich cultural resources of celebrities; Fuzhou has obvious characteristics of dramatic culture resources with Tang Xianzu' s well known for opera at home and abroad and Fuzhou tea-picking opera and Yihuang opera having their own features. With good ecological environment, Fuzhou ranked first in "Chinese Oxygen Bar" assessment in China's mainland. Dajue Mountain scenic area has beautiful scenery. Ancient Liukeng Village of Le' an County is called as a thousand-year-old village. Tourism resources in both Nanchang and Fuzhou are different and highly complementary.

\section{Easy Transportation in Both Nanchang and Fuzhou}

Multiple transport lines in waterway, railway and highway are very convenient such as Xiangtang-Putian Railway, Fuzhou-Yinchuan Expressway, National Highway 106 and Ganjiang River-Fuhe River, etc. Xiangtang-Putian traffic corridor has become an important intercity traffic corridor in Jiangxi Province connecting Nanchang and Fuzhou. As the high-speed railway between Nanchang and Fuzhou opens, it takes only 40min of driving time between both place. Thus the transport in Nanchang and Fuzhou is more convenient and faster.

\section{Complementary advantages in tourist \& tourism resources of Nanchang and Fuzhou}

Nanchang is a provincial city with a large population. It is also an important place to deliver passengers and tourist generating region. With rich culture tourism resources and complete highway and waterway transport, Fuzhou is highly competitive. But, Fuzhou has no airports and air route for the time being, the passengers who take a plane shall be delivered by Changbei International Airport, so Nanchang, the provincial capital, has become an important transit area for Fuzhou's connecting overseas tourists.

\section{Issues existing in integrative development of tourism of Nanchang-Fuzhou}

\section{Low integration degree of tourism resources in Nanchang and Fuzhou}

Fuzhou has abundant cultural resources, especially celebrity tourism resources, such as Tang Xianzu's good reputation at home and abroad and high popularity of mountain and water resources 
of Dajue Mountain. These resources are very different from red tourism resources in Nanchang, which are complementary. But the tourism resources were not integrated during development to increase integration effects, which greatly influences enhancement of tourism resources value of Nanchang- Fuzhou. Red tourism of Nanchang is mainly subject to visiting, in which the tourists stay a shorter time in Nanchang. Red tourism and other tourism resources in Nanchang have low integration degree. Cultural relics tourism and ecological tourism of Fuzhou and red tourism of Nanchang generate no well effective interaction. Regional tourism products are still in sightseeing phase, resource development is in superficial level and tourism brand which is influential in China and even the world is not created yet and is not in the primary stage of integration development due to low integration degree of tourism resources in both cities and integration still in initial stage.

\section{Insufficient propaganda in tourism of Nanchang-Fuzhou and not doing well in establishment of brand together}

Both Nanchang City and Fuzhou City run a marketing campaign for tourism separately and stress advantages and features of tourism respectively in tourist propaganda and there are also problems existing in their own publicity. Without good joint propaganda, join forces and sufficient propaganda work in tourism marketing. Forming no clear and unified tourism image, and the efforts taken to build overall tourism image is not enough. Not doing a good job in establishment of brand and entirely highlighting the advantages and features of tourism and then advertising it externally. Not carrying out tourism publicity from multiple point of view and multi-mode in tourism publicity, and forming no integrative project in marketing for foreign publicity. Making little use of new medium in means of tourism propaganda and not fully use network techniques for tourism propaganda.

\section{Lack of scientific plan in tourism of Nanchang and Fuzhou and not giving full play of leading role of the government}

Both Nanchang and Fuzhou don't establish good coordinated mechanisms due to restriction of administrative division. Both cities randomly develop and use their own tourism resources, which leads to poor reflection of characteristics of tourism resources and no good formation of complete tourism product system. Both Nanchang and Fuzhou don't give full play to leading role of government and are lack of reasonable planning due to restriction of administrative division, which results in repeated construction and not scientific and reasonable allocation of tourism resources and resource wastes. Lack of reasonable planning in tourism integration, both Nanchang and Fuzhou don't use the concept of planning first to lead scientific and reasonable development of overall tourism, carry out unified planning in tourism routes, tourism products, tourism industry and tourism resources, etc. Not knowing how to solve problems such as how to make up the shortfall, how to support advantages for development, how to make clear positioning and how to seek breakthroughs in points, etc.

\section{Further improvement required for infrastructure construction and service quality of Nanchang and Fuzhou}

It has achieved certain developments in infrastructure construction and service quality of two administrative zones of both Nanchang and Fuzhou, but the level of tourism transport which connects the tourist source region with scenic spots in Nanchang-Fuzhou is not so high, and the transportation accessibility shall be strengthened, all these factors influence integration of tourism products and design and arrangement of tourist routes in both Nanchang and Fuzhou area. In addition, the infrastructures which is involved in food, housing, transportation and travel, etc. for tourists in Nanchang-Fuzhou area and service level for tourism service are not perfect, thus affecting integrative development of tourism in Nanchang-Fuzhou, even Jiangxi Province. 


\section{Solutions to integrative development of regional tourism of Nanchang-Fuzhou}

\section{Accelerate integration of regional tourism resources and make joint endeavor to promote first-rate tourism routes}

It is necessary for Nanchang-Fuzhou to accelerate resources integration of its regional tourism and foster a robust atmosphere from its integrative development. Regarding rich tourism resources of Nanchang-Fuzhou complementary to one another, Nanchang and Fuzhou should make best use of individual regional tourism advantages for integrative development of regional tourism. To this end, it is required that a sizable tourism and a great development be considered and Nanchang-Fuzhou tourism markets be integrated for a united brand and an overall image of its tourism. In the development of tourism, Nanchang and Fuzhou should objectively and fully recognize the overall features of Nanchang-Fuzhou and respective regional features for optimized integration of both of tourism resources. On one hand the integrative development features its advantages so that tourism products in different themes will be developed; on the other hand the tourism advantages of one another balance respective disadvantages so that a collection of specialized united first-rate tourism scenery and routes will be integrated for playing a shared and complementary role in tourism resources of Nanchang-Fuzhou. A multilevel and multi-functional tourism network of Nanchang-Fuzhou should be established through acceleration of tourism resources integration.

\section{Enhance united marketing and build up a well-received brand image}

There are similarities shared by Nanchang and Fuzhou in terms of tourism products and tourism development. In the past, the two cities publicized tourism in an individual and competitive manner, leading to a waste of resources and a weakened core competitive edge of tourism products of Nanchang and Fuzhou. The two cities separately marketed and publicized tourism, and led to bad-effect competition, making tourists source captured, tourism scale diminished and overall competitive edge lowered. Brand-new and well-received regional overall image can combine separate product images and attraction of relevant brands, fostering a huge cohesive force [7]. Therefore, the integrative development of regional tourism of Nanchang-Fuzhou requires scientific layout and brand establishment for improving tourism image of Nanchang-Fuzhou. The establishment of tourism brand image helps to improve the fame of tourism destinations, tourism attraction and competitive edge of Nanchang-Fuzhou. The tourism image of Nanchang-Fuzhou embodies refinement and epitome of tourism products of Nanchang-Fuzhou. In case that previous problems arising in the past repeat inside Nanchang-Fuzhou tourism region, the image of the whole region should be well planned, the tourism features and development advantages should be clearly known and tourism functional locations of every part should be recognized for balancing advantages and disadvantages and collaborating regional development. For instance, Nanchang specializes in revolutionary tourism and urban leisure tourism while Fuzhou specializes in cultural tourism and ecological tourism. On top of developing its specialized tourism inside the region, Nanchang-Fuzhou should develop an extra series of tourism routes with clear themes, rich meanings and sharp features, emphatically publicize a collection of brand tourism products of nationwide influence, establish a national tourism center of ecology, culture, leisure and historic revolution, nurture its distinct tourism economy belt and facilitate its integrative development of tourism. Nanchang-Fuzhou should make use of its tourism videos and tourism manuals for marketing, and combine new media and network information technology to publicize specialized tourism resources along Xiangtang-Putian Railway Corridor from different perspectives and through different ways for establishing a brand-new tourism image of Nanchang-Fuzhou. Nanchang-Fuzhou should enhance the tourism publicity, strengthen its international brand for the tourism image of culture, ecology and leisure through international tourism introduction fairs.

\section{Dominated by the government and depend upon scientific layout}

First and foremost, the Jiangxi provincial government should play its dominant role in tourism integration. In the backdrop of and at the premise of economic globalization, it will be hard for 
either region of Nanchang-Fuzhou to make achievements when confronted with the environment and background. The government plays the most effective controlling role in the regional development. Its policies and controls influence local tourism development and directly relate to benefit coordination of trans-regional administration division and trans-administration level. It's unimaginable to integrate regional tourism without government's coordination [8]. Therefore, the government plays a significant role in such background. The government should play its dominant role in Nanchang-Fuzhou integrative development of tourism and establish effective cooperation and coordinative mechanism between governments.

Secondly, scientific layout shall be prioritized in the Nanchang-Fuzhou integrative development of tourism. The tourism resources of the two cities should be integrated for promoting the Nanchang-Fuzhou integrative development of tourism. The integration of tourism resources is based on tourism planning which is the direction and blueprint of tourism development of the two cities. The tourism planning of Nanchang-Fuzhou should start higher and achieve better in a paramount perspective and on a long-term footing. Nanchang and Fuzhou municipal governments should take lead in formulating authoritative Nanchang-Fuzhou Tourism Integration Planning in terms of uniformly plan the tourism products, tourism industry and tourism routes to promote the integrative development of regional tourism of Nanchang-Fuzhou. The integration of tourism markets will be realized through incorporation of Nanchang-Fuzhou tourism group companies, development of tourism enterprises and collaboration of enterprises in Nanchang-Fuzhou.

\section{Improve tourism infrastructure and tourism service quality}

As a prerequisite of regional economic development, infrastructure almost closely connects with processes of production and daily life in the development of social economy. Therefore, infrastructure construction and development plays a crucial role in regional economic development [9]. It is required that Nanchang-Fuzhou integrative development of regional tourism be based on bettered tourism infrastructure. Therefore, tourism infrastructure should be bettered. Traffic plays a basic role in sub-system operation of internal motivity. Traffic integration is the prerequisite and base of facilitating regional tourism integration [10]. Although the traffic system of Nanchang-Fuzhou continues to improve, the integrative development has not yet been formed in whole, which affects its coordinated configurat on and sharing of regional tourism and its development of regional economic integration. In adherence to regional integration, the traffic network construction should be strengthened in major cities such as Nanchang, Fuzhou, Jiujiang, Shangrao, etc. Accordingly, Nanchang-Fuzhou traffic integration and traffic network will be formed, enhancing tourism accessibility to Nanchang-Fuzhou and boosting the economy in Jiangxi Province. The construction of Fuzhou airport and intercity railway, intercity highway and waterway of Nanchang-Fuzhou should be accelerated and a vertical traffic of waterway, highway and airway of Nanchang-Fuzhou should be established, making highway, railway and waterway matched in a seamless manner. Meanwhile, Nanchang-Fuzhou should be centered on Tengwang Pavilion, Dajue Mountain and revolutionary tourism scenery, strengthen tourism infrastructure construction and management such as supermarkets, travel agencies and hotels, improve tourism service quality, facilitate seamless match of catering, accommodation, traffic, travel and shopping and enhance overall competitive edge of tourism enterprises in Nanchang-Fuzhou.

\section{References}

[1] Geng Yuhai. Improvement of Cooperation Mechanism and Realization of Integrative Development of Regional Tourism in the Yangtze Delta [J]. Chinese Consulting Engineers, 2007(9):27-28.

[2] Analysis on the Innovating of the Tourism Integration Mechanism in the Pearl River Delta [J]. Geography and Geo-information Science, 2001(6):96-100

[3] Ding Min. The Yangtze Delta Region Traveling Integration Research [J]. Market Weekly, 
2006(11):58-59.

[4] Wu Bihu. Fundamentals of Regional Tourism Planning [M]. Beijing: China Travel \& Tourism Press, 2001.

[5] Yang Hong, Luo Qiujun et al, Study on Changsha-Zhuzhou-Xiangtan Tourism Integration [J]. Tropical Geography, 2003 (12).

[6] Wan Ke, Huang Jianxin et al. Development of Tourism Integration in Nanchang-Jiujiang Area [J]. Economic Geography, 2016 (3).

[7] Luo Xiaobin, Fang Shimin. Insight into Integration of Regional Red Tourism Brand [J]. Journal of Huaihua University, 2006, 25 ( 3) : 62 - 65.

[8] Ge Licheng, Nie Xianzhong and Li Wenfeng. Study on Tourism Integration in Yangtze River Delta region [J] Zhejiang Social Sciences, 2006 (2).

[9] Sun Jiuwen. Regional Economics [M]. Beijing: China Renmin University Press, 2010.

[10] Huang Hongjie, Li Kanwei. Advantages, Bottleneck and Countermeasures in Development of Tourism Integration in Circum-Bohai-sea Region [J]. Enterprise Economy, 2010 (10): 157-159. 